\title{
How to Set Up Your STEM for EELS at Very High Energy Losses
}

\author{
Ian MacLaren ${ }^{1}$, Alan J. Craven ${ }^{1}$, Colin Black ${ }^{1}$, Sam McFadzean $^{1}$ and Hidetaka Sawada ${ }^{2}$ \\ 1. School of Physics and Astronomy, University of Glasgow, Glasgow G12 8QQ, UK \\ 2. JEOL UK Ltd., JEOL House, Silver Court, Watchmead, Welwyn Garden City, Herts. AL7 1LT, UK
}

Whilst EELS is typically performed on a (scanning) transmission electron microscope with a postcolumn filter, relatively little has been published recently on optimizing the coupling between the two. In order to work well with modern aberration corrected STEMs, where the convergence semi-angle of the probe is likely to be of the order of $30 \mathrm{mrad}$, a large acceptance angle is required at the spectrometer. This requires that a rather small camera length is used in the microscope to compress the whole bright field disc inside the acceptance aperture. For example, the camera length typically used for EELS in the JEOL ARM200F used in this work is just $2 \mathrm{~cm}$. Most of the camera lengths for such a microscope are provided by lens settings optimized for the best quality imaging at the beam energy, and the performance of these lens settings at higher energy losses may not have been considered in detail.

Nevertheless, this issue of the optimum coupling of a STEM to a post-column spectrometer has been considered previously by Craven and Buggy [1] for a very simple system of just two post-specimen lenses. In that work, they showed that the collection angle into the spectrometer varied much less drastically with energy loss for a mode in which the first post-specimen lens provided an image that served as a virtual object to the second lens, rather than one in which the first lens provided a real image to form a real object for the second lens. This concept was used in the more complex layout of the ARM200F, where there are four post-specimen lenses, and settings were calculated using a mixture of virtual and real objects for the lenses. A careful analysis of the effects of the different settings was performed by analyzing the optical performance of the imaging system and by observing how the STEM diffraction pattern varied with the electron energy in the range 197 and $203 \mathrm{keV}$ (using the EFTEM mode, which leaves the lens excitations unaltered). It is shown (Figure 1) that an optimized setting gives a much more constant camera length over the energy loss range $0-3 \mathrm{keV}$. The cut-off at the edge of the pattern is now from the differential pumping aperture and not from fold-over of high angle rays due to aberrations in the post specimen lenses, as was the case with the original $2 \mathrm{~cm}$ camera length.

The result of this is that the reduced fold-over of the high angle scattering into the intensity stripe on the camera that is integrated into the spectrum gives a much better behaved background as shown in Figure 2. This figure shows background-subtracted Fourier-ratio deconvoluted spectra of the Mo- $\mathrm{L}_{2,3}$ edge from $\mathrm{MoO}_{2}$ and shows that the optimised camera length gives much better performance over a range of thicknesses, whereas the original camera length results in the incorporation of some thickness-dependent background which is not elemental specific information belonging to the Mo edge.

The use of this new advance in microscope-spectrometer coupling gives improved results at $200 \mathrm{keV}$ for all edges above about $2 \mathrm{keV}$. The limits of this setting have been explored in detail and it is found that the performance gradually deteriorates with increasing energy loss until the EELS stripe collapses to a minimum thickness at about $9.2 \mathrm{keV}$. This corresponds to a crossover appearing at the entrance aperture of the spectrometer giving a zero camera length and a collection angle limited by the differential pumping aperture. Near to this energy loss, the EELS edge shapes are less well-defined and there is more background disturbance. Nevertheless, edges such as Ti, Mn and Fe K-edges have been acquired 
before this crossover. Moreover, a detailed study of the effects of oxidation on the $\mathrm{Sn}-\mathrm{L}_{2,3}$ edge will be presented, which is consistent with previous X-ray absorption spectroscopy studies of the same edge. Finally, after this $9.2 \mathrm{keV}$ crossover, the $\mathrm{Ta}_{3}$ and $\mathrm{L}_{2}$ edges have been recorded at $\sim 9.8$ and $11 \mathrm{keV}$.

Whilst one application of this work is clearly to extend the range of EELS investigations to higher losses at $200 \mathrm{keV}$, this work is also key at lower accelerating voltages (e.g. 60 or even $30 \mathrm{keV}$ ) that are required for materials with low knock-on damage thresholds. At such voltages, the chromatic effects in the post-specimen lenses are correspondingly larger. Thus even reaching a $1 \mathrm{keV}$ energy loss without the effects described above is currently difficult. However, it will be considerably simpler if these approaches are adopted in the choice of the lens settings or even the design of the lens system itself.

References:

[1] A. J. Craven and T. W. Buggy, Ultramicroscopy 7 (1981) p. 27.
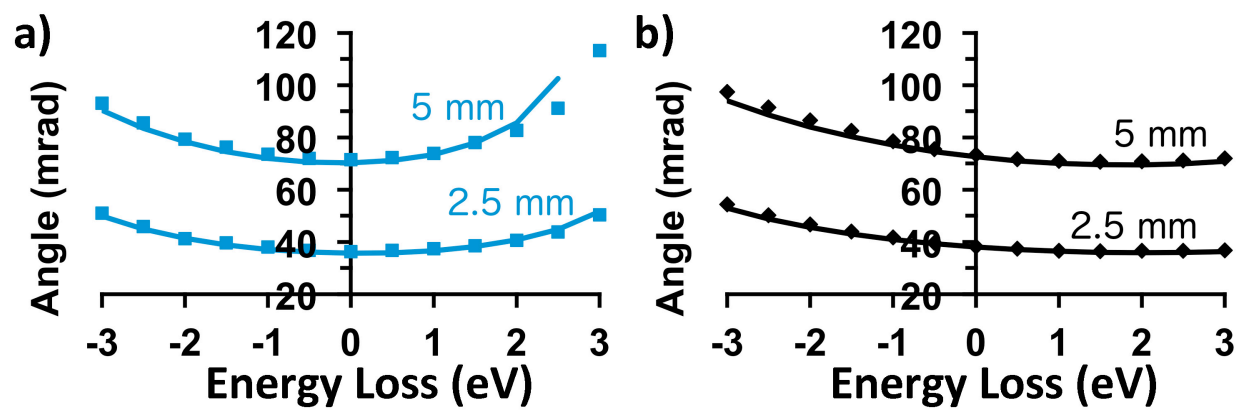

Figure 1. Acceptance angle of the spectrometer as a function of energy loss: a) Original $2 \mathrm{~cm}$ camera length; b) optimised $2 \mathrm{~cm}$ camera length giving a much more constant acceptance angle over the energy range of most commonly used edges. Results for both $2.5 \mathrm{~mm}$ and $5 \mathrm{~mm}$ entrance apertures are shown.

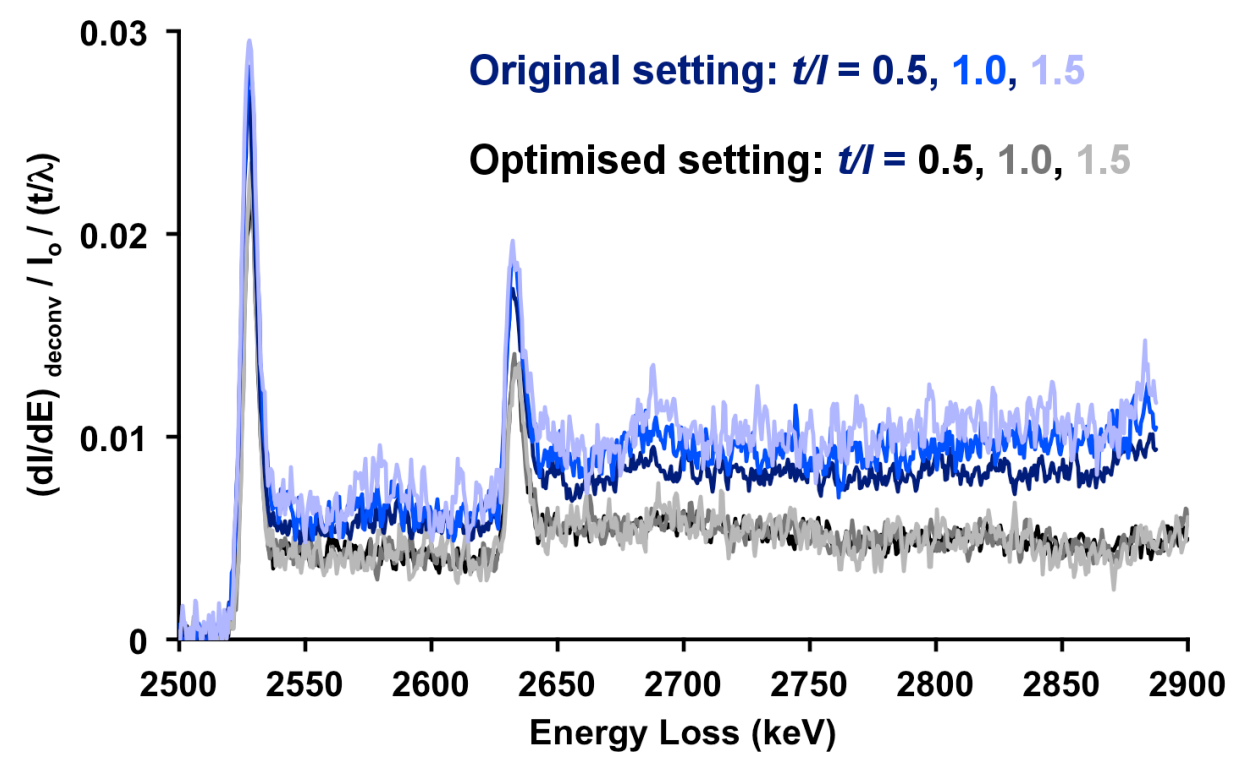

Figure 2. The Mo- $\mathrm{L}_{2,3}$ edge from $\mathrm{MoO}_{2}$ after background subtraction and Fourier-ratio deconvolution. Blue spectra are from the original $2 \mathrm{~cm}$ camera length, and grey spectra are from the modified $2 \mathrm{~cm}$ setting with improved performance over the 0-3 $\mathrm{keV}$ range. Three thicknesses were used for each setting, $t / \lambda=0.5,1.0$ and 1.5 (dark, mid and light blue for original $2 \mathrm{~cm}$, and dark, mid and light grey for the optimized $2 \mathrm{~cm}$ camera length). 